\title{
Inhibition of the Chloramphenicol-induced Alternative Oxidase-Mediated Ubiquinol-15 Oxidation in Neurospora crassa Mitochondria by 2-Thenoyltrifluoroacetone
}

\author{
By JOS VANDERLEYDEN, ${ }^{1}$ HUBERT VERACHTERT ${ }^{1 *}$ AND \\ HELMUT BERTRAND ${ }^{2}$ \\ ${ }^{1}$ Laboratory for Industrial Microbiology and Biochemistry, University of Leuven, \\ 3030 Heverlee-Louvain, Belgium \\ ${ }^{2}$ Department of Biology, University of Regina, Saskatchewan, Canada S4S 0A2
}

(Received 1 December 1980; revised 29 January 1981)

\begin{abstract}
Ubiquinol-15 can be used as an electron donor for both the conventional cytochrome chain and the chloramphenicol-induced alternative pathway of Neurospora crassa mitochondria. The oxidation of ubiquinol-15 through the chloramphenicol-induced cyanide-insensitive pathway is not inhibited by antimycin A and is strongly inhibited by salicylhydroxamic acid. In contrast, the oxidation of duroquinol by $N$. crassa mitochondria is sensitive to antimycin A under all circumstances, except for some portion which is believed to be a non-enzymic autoxidation reaction. 2-Thenoyltrifluoroacetone, a specific inhibitor of the succinateubiquinone oxidoreductase activity of the respiratory chain, was found to be a potent inhibitor of the chloramphenicol-induced alternative oxidase-mediated NADH and ubiquinol15 oxidation in $N$. crass a mitochondria. It may be concluded that 2-thenoyltrifluoroacetone interacts with a specific electron carrier of the alternative oxidase, possibly an iron-sulphur centre associated with an ubisemiquinone.
\end{abstract}

\section{INTRODUCTION}

Organisms with cyanide-insensitive respiration have a branched respiratory chain (Palmer, 1976; Solomos, 1977). One branch is the conventional cytochrome chain. The second branch is of unknown composition and is called the alternative respiratory pathway or alternative oxidase. It is generally accepted that ubiquinone functions as the branchpoint for the two pathways (Hanssens et al., 1978; Huq \& Palmer, 1978a; Rich \& Bonner, 1978). The alternative oxidase is insensitive to cyanide and antimycin $A$, the classical inhibitors of cytochrome-mediated respiration, but can be specifically inhibited by substituted hydroxamic acids such as salicylhydroxamic acid (Schonbaum et al., 1971).

Various attempts have been made to isolate the mitochondrial component which catalyses the $\mathrm{O}_{2}$-reducing step of the alternative oxidase. Huq \& Palmer $(1978 b)$ reported the partial purification by ion-exchange chromatography of a fraction of lubrol-solubilized cyanideinsensitive mitochondria of Arum maculatum, which promoted salicylhydroxamic acidsensitive oxidation of duroquinol. Rich (1978) used deoxycholate to solubilize cyanideinsensitive mitochondria of $A$. maculatum, and obtained a preparation that showed salicylhydroxamic acid-sensitive oxidation of menadiol and ubiquinol-5. In both cases, however, there were some complications, and the possibility that the activity was due to autoxidation rather than an enzyme-catalysed oxidation of the quinols could not be excluded. Moreover, the autoxidation of duroquinol and menadiol is inhibited by salicylhydroxamic acid, and therefore salicylhydroxamic acid sensitivity is not sufficient as a criterion to 
distinguish simple autoxidation from enzyme-catalysed oxidation of quinols (Vanderleyden et al., 1980a).

Huq \& Palmer (1978c) demonstrated that duroquinol is oxidized in cyanide-insensitive mitochondria through the pathway which is antimycin A- and cyanide-insensitive and salicylhydroxamic acid-sensitive. However, Von Jagow \& Bohrer (1975) reported that the oxidation of duroquinol in Neurospora crassa mitochondria possessing the alternative pathway is cyanide-insensitive but antimycin A-sensitive. Therefore, duroquinol is thought to react with cytochrome $b$ in $N$. crassa, as was found in mitochondria of animal tissues (Slater et al., 1961; Boveris et al., 1971). Hence, duroquinol is not a suitable substrate for the alternative oxidase of $N$. crassa since cytochrome $b$ is not an intermediate of the $\mathrm{O}_{2}$-reducing part of the alternative oxidase, and its use in the study of the alternative oxidase so far is restricted to plant mitochondria.

In this paper we describe the use of ubiquinol-15 as an electron donor to both the cyanide-sensitive and cyanide-insensitive pathway of $N$. crassa. In addition, we report that 2-thenoyltrifluoroacetone, earlier described as a specific inhibitor of the succinate-ubiquinone region of the respiratory chain (Takemori \& King, 1964), is a potent inhibitor of the alternative oxidase-mediated oxidation of NADH and ubiquinol-15 in $N$. crassa mitochondria.

\section{METHODS}

Micro-organism and growth. The organism used was Neurospora crassa wild type, 74-OR23-1A (abbreviated 74A), obtained from the Fungal Genetics Stock Center (FGSC; Humboldt State University Foundation, Arcata, Calif., U.S.A.). Mycelium was grown at room temperature in liquid Vogel's medium (Vogel, 1964) as described previously (Bertrand et al., 1968; Bertrand \& Pittenger, 1969). Non-induced (cyanide-sensitive) mycelium was obtained after growth for $18 \mathrm{~h}$ in the standard Vogel's medium. Induced (cyanide-insensitive) mycelium was obtained after growth for $48 \mathrm{~h}$ in Vogel's medium supplemented with chloramphenicol $\left(5 \mathrm{mg} \mathrm{ml}^{-1}\right)$.

Preparation of mitochondria. Mycelium was disrupted with a grinding mill (Weiss et al., 1970), and mitochondria were prepared as previously described (Bertrand \& Pittenger, 1969).

Reduction of ubiquinone-15. Ubiquinone-15 was reduced using the method of Rieske (1967), except that $\mathrm{NaBH}_{4}$ was used instead of $\mathrm{Na}_{2} \mathrm{~S}_{2} \mathrm{O}_{4}$.

$\mathrm{O}_{2}$ consumption. $\mathrm{O}_{2}$ consumption of mitochondria was measured polarographically with a Clark-type oxygen electrode. Unless otherwise stated, the reaction mixture consisted of $0.44 \mathrm{M}$-sucrose, $10 \mathrm{~mm}$-Tris $/ \mathrm{HCl}$,

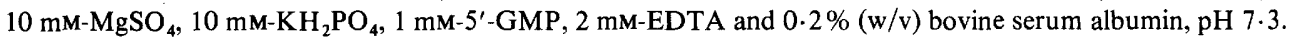

Protein determination. Mitochondrial protein was determined by the biuret method (Gornall et al., 1949) with modifications to account for absorbance due to turbidity of the sample (Busenherz et al., 1953).

Ubiquinol-15 concentration. Ubiquinol-15 was made up in ethanol and the concentration was determined as described previously (Vanderleyden et al., 1980c).

Materials. 5'-GMP was obtained from Sigma. 2-Thenoyltrifluoroacetone was obtained from Fisher Scientific Co., Fairlawn, N.J., U.S.A., and stored as a $500 \mathrm{~mm}$ solution in ethanol. Duroquinol was obtained from Pfaltz \& Bauer, Stamford, Conn., U.S.A., and made up fresh as a $300 \mathrm{~mm}$ solution (determined gravimetrically) in ethanol. Ubiquinone-15 was a generous gift of Hoffman-la-Roche, Basel, Switzerland. The purity of the sample was checked by reversed-phase thin-layer chromatography (Ramasarma \& Jayaraman, 1971).

\section{RES ULT S}

\section{Succinate and duroquinol oxidase activities of induced mitochondria of $N$. crassa}

The succinate and duroquinol oxidation in induced mitochondria of $N$. crassa are compared in Fig. 1. Induced mitochondria contained both the conventional cytochrome chain and the alternative pathway, although the former was present to a lesser extent than in non-induced mitochondria due to inhibition of the synthesis of cytochrome $b$ and cytochrome $a a_{3}$ by chloramphenicol. The oxidation of duroquinol was insensitive to cyanide but sensitive to antimycin A, whereas the oxidation of succinate was largely insensitive to both inhibitors and to the same extent. 


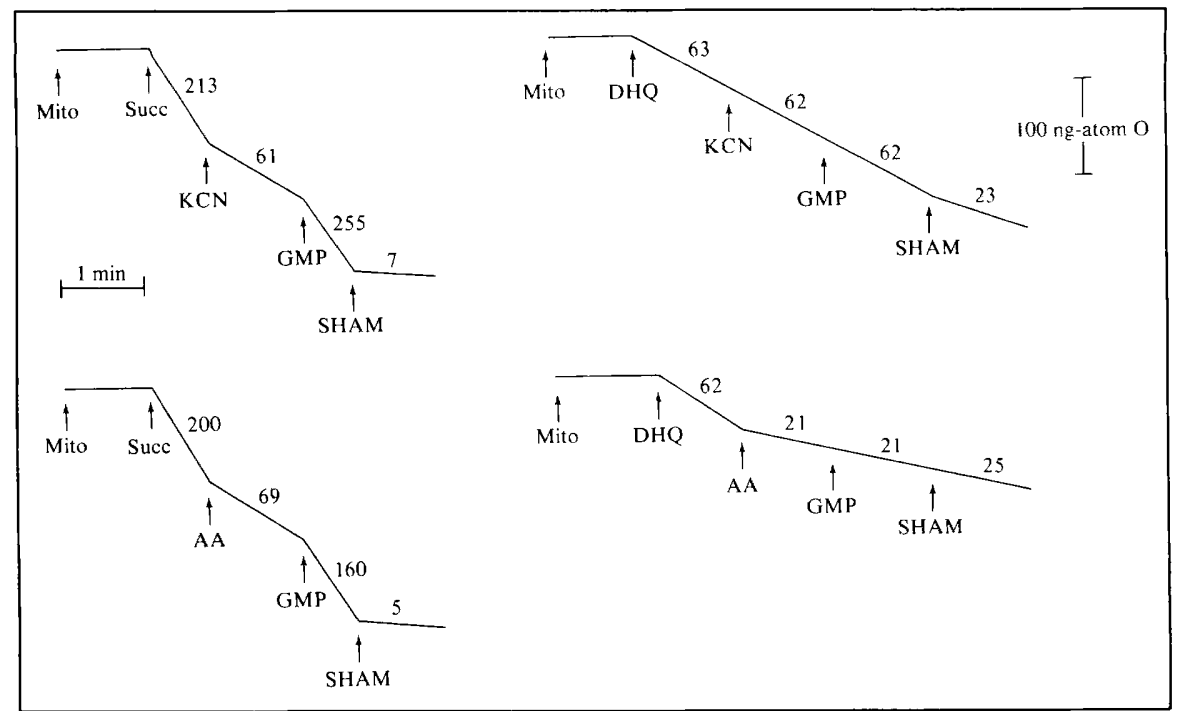

Fig. 1. Succinate oxidase (left) and duroquinol oxidase (right) activities of induced mitochondria of Neurospora crassa. The oxidase activities were measured polarographically with succinate (Succ) or duroquinol (DHQ) as substrate. The reaction mixture (final volume $3 \mathrm{ml}$, temperature $25^{\circ} \mathrm{C}, \mathrm{pH} 7 \cdot 3$ ) consisted of $0.3 \mathrm{M}$-sucrose, $10 \mathrm{mM}-N$-tris(hydroxymethyl)methyl-2-aminomethane sulphonic acid, $5 \mathrm{mM}-\mathrm{KH}_{2} \mathrm{PO}_{4}, 5 \mathrm{mM}-\mathrm{MgCl}_{2}$. At the times indicated, additions were made of mitochondria (Mito) $(0.25$ to $0.6 \mathrm{mg}$ protein), $1 \mathrm{mM}-\mathrm{KCN}, 3 \mu \mathrm{g}$ antimycin A (AA), $1 \mathrm{mM}-5^{\prime}-\mathrm{GMP}$ (GMP) and $2.6 \mathrm{~mm}$ salicylhydroxamic acid (SHAM). The numbers above the traces represent the rates of $\mathrm{O}_{2}$ consumption in $\mathrm{ng}$-atom $\mathrm{O} \mathrm{min}^{-1}(\mathrm{mg} \text { protein })^{-1}$.

5'-GMP ( $1 \mathrm{mM})$ was included in the oxidase assays, because the alternative oxidase activity of $N$. crassa mitochondria is stimulated by 5'-GMP (Vanderleyden et al., 1980 b). These experiments agreed with the results of Von Jagow \& Bohrer (1975). The residual oxidation rate with duroquinol, in the presence of both salicylhydroxamic acid and cyanide or antimycin $\mathrm{A}$, must have been due to a non-specific oxidation, including autoxidation, since it was not found with succinate or NADH (not shown).

Ubiquinol-15 as an electron donor for the respiratory pathways of $N$. crassa

The oxidation rates of non-induced and induced mitochondria of $N$. crassa with NADH, succinate and ubiquinol-15 as substrates were tested for sensitivity to cyanide, antimycin A and salicylhydroxamic acid. Non-induced mitochondria have only the conventional cytochrome respiratory pathway. Table 1 shows that the response to the different inhibitors of both the alternative oxidase-mediated and the cytochrome oxidase-mediated ubiquinol-15 oxidase activities was very similar to the response of the corresponding NADH and succinate oxidase activities. It is important to note that the oxidation of ubiquinol-15 by induced mitochondria was almost completely insensitive to cyanide as well as antimycin A, and to the same extent. Hence ubiquinol-15 is a more suitable quinol substrate for the alternative oxidase of $N$. crassa mitochondria than is duroquinol, since duroquinol oxidation in induced mitochondria is sensitive to antimycin A. The complete inhibition of the cytochrome oxidase-mediated ubiquinol-15 oxidation by cyanide or antimycin $\mathrm{A}$ was clear proof that there was no interference from non-enzymic oxidation reaction of the substrate, an observation in agreement with the properties of ubiquinol-15 described by Futami et al. (1979).

The amounts of mitochondrial protein used to determine the NADH and succinate oxidation rates were, respectively, 10 and 20 times higher than the amount used to determine the ubiquinol-15 oxidation rates (see legend of Table 1). When ubiquinol-15 is used as an 
Table 1. Inhibitor sensitivities of the oxidase activities of non-induced and induced mitochondria of Neurospora crassa

The oxidase activities were measured polarographically with $1 \mathrm{~mm}-\mathrm{NADH}, 10 \mathrm{~mm}$-succinate or $1 \mathrm{~mm}$-ubiquinol-15 as substrates. The reaction mixture (final volume $3 \mathrm{ml}$, temperature $25^{\circ} \mathrm{C}$, $\mathrm{pH} \mathrm{7.3)} \mathrm{consisted} \mathrm{of} 0.44 \mathrm{M}$-sucrose, $10 \mathrm{~mm}$-Tris/ $\mathrm{HCl}, 10 \mathrm{~mm}-\mathrm{KH}_{2} \mathrm{PO}_{4}, 10 \mathrm{~mm}-\mathrm{MgSO}_{4}, 2 \mathrm{~mm}$-EDTA, $0.2 \%(\mathrm{w} / \mathrm{v})$ bovine serum albumin and $1 \mathrm{~mm}-5^{\prime}-\mathrm{GMP}$. The amounts of mitochondrial protein used were $0.3 \mathrm{mg}$ for the NADH oxidase assays, $0.6 \mathrm{mg}$ for the succinate oxidase assays, and $0.03 \mathrm{mg}$ for the ubiquinol-15 oxidase assays. The original activities [ng-atom $\mathrm{O} \mathrm{min} \mathrm{m}^{-1}(\mathrm{mg} \text { protein) })^{-1}$ ] of the different assays were $(a)$ 197, (b) 177, (c) 195, (d) 410, (e) 396 and $(f)$ 480. Abbreviations: SHAM, salicylhydroxamic acid; Succ, succinate; UQ-15, ubiquinol-15.

\begin{tabular}{|c|c|c|c|c|c|c|}
\hline \multirow[b]{3}{*}{ Inhibitor } & \multicolumn{6}{|c|}{ Percentage inhibition of maximal rate } \\
\hline & \multicolumn{3}{|c|}{ Non-induced mitochondria } & \multicolumn{3}{|c|}{ Induced mitochondria } \\
\hline & $\begin{array}{c}(a) \\
\mathrm{NADH}\end{array}-\mathrm{O}_{2}$ & $\begin{array}{c}(b) \\
\text { Succ-- }{ }_{2}\end{array}$ & $\begin{array}{c}(c) \\
\text { UQ-15- }\end{array}$ & $\begin{array}{c}(d) \\
\mathrm{NADH}\end{array}-\mathrm{O}_{2}$ & $\begin{array}{c}(e) \\
\text { Succ-O }\end{array}$ & $\begin{array}{c}(f) \\
\text { UQ-15-O }\end{array}$ \\
\hline Tone & 0 & 0 & 0 & 0 & 0 & 0 \\
\hline $\mathrm{CN}(1 \mathrm{mM})$ & 100 & 100 & 98 & 7 & 8 & 8 \\
\hline Intimycin $\mathrm{A}(3 \mu \mathrm{g})$ & 100 & 100 & 98 & 11 & 10 & 8 \\
\hline HAM $(2.6 \mathrm{~mm})$ & 0 & 0 & 0 & 87 & 80 & 80 \\
\hline $\begin{array}{l}\operatorname{HAM}(2.6 \mathrm{mM})+ \\
\mathrm{KCN}(1 \mathrm{mM})\end{array}$ & 100 & 100 & 98 & 95 & 86 & 98 \\
\hline
\end{tabular}

\section{Table 2. Effects of 2-thenoyltrifluoroacetone on the oxidase activities of non-induced and induced mitochondria of Neurospora crassa}

The oxidase activities were measured polarographically as indicated in the legend of Table 1 . Cytochrome-mediated oxidase activities were measured with non-induced mitochondria, and alternative oxidase-mediated activities with induced mitochondria. The reaction medium for the oxidase assays was the same as indicated in the legend of Table 1 , except that $1 \mathrm{mM}-\mathrm{KCN}$ or $3 \mu \mathrm{g}$ antimycin $\mathrm{A}$ was included in the medium for the alternative oxidase-mediated activities. The original activities of the different assays were of the same order of magnitude as those reported in the legend of Table 1 . Abbreviations: TTFA, 2-thenoyltrifluoroacetone; SHAM, salicylhydroxamic acid; Succ, succinate; UQ-15, ubiquinol-15.

\begin{tabular}{|c|c|c|c|c|c|c|}
\hline \multirow[b]{3}{*}{ Inhibitor } & \multicolumn{6}{|c|}{ Percentage inhibition of maximal rate } \\
\hline & \multicolumn{3}{|c|}{ Non-induced mitochondria } & \multicolumn{3}{|c|}{ Induced mitochondria } \\
\hline & $\mathrm{NADH}-\mathrm{O}_{2}$ & Succ- $-\mathrm{O}_{2}$ & UQ-15- $\mathrm{O}_{2}$ & $\mathrm{NADH}-\mathrm{O}_{2}$ & Succ- $-\mathrm{O}_{2}$ & UQ-15- $\mathrm{O}_{2}$ \\
\hline None & 0 & 0 & 0 & 0 & 0 & 0 \\
\hline TTFA (1 mM) & 0 & 77 & 0 & 88 & 82 & 95 \\
\hline $\begin{array}{l}\text { TTFA }(1 \mathrm{mM})+ \\
\mathrm{KCN}(1 \mathrm{mM})\end{array}$ & 100 & 100 & 95 & 88 & 82 & 95 \\
\hline $\begin{array}{l}\text { TTFA }(1 \mathrm{mM})+ \\
\quad \text { SHAM }(2.6 \mathrm{mM})\end{array}$ & 0 & 75 & 0 & 92 & 100 & 100 \\
\hline
\end{tabular}

exogenous electron donor, only a small fraction of the ubiquinol-15 added to the reaction vessel $(1 \mathrm{mM})$ is soluble in water. Thus the actual concentration of ubiquinol-15 in the respiration vessel was unknown, and saturation kinetics could only be measured by adjusting the amount of mitochondrial protein with the given amount of water-soluble ubiquinol-15. In these experiments, saturation kinetics were obtained with $1 \mathrm{~mm}$-ubiquinol-15 added and $30 \mu \mathrm{g}$ mitochondrial protein.

Inhibition of the alternative oxidase activity of $N$. crassa by 2-thenoyltrifluoroacetone

2-Thenoyltrifluoroacetone is known to be a potent inhibitor of succinate oxidation in membrane preparations from animal and plant tissues, fungi and bacteria, acting at the 


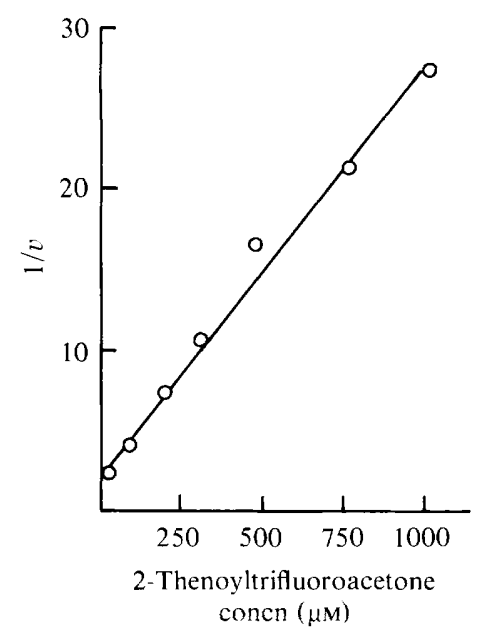

Fig. 2. Dixon plot (Dixon, 1953) of the inhibition of the alternative oxidase-mediated ubiquinol-15 oxidation in induced mitochondria of Neurospora crassa by 2-thenoyltrifluoroacetone. The oxidase activities were measured polarographically as indicated in the legend of Table 1 . The reaction mixture for the assay was the same as indicated in the legend of Table 1 , except that $1 \mathrm{mM}-\mathrm{KCN}$ was also included. Oxidase activities were measured with $0.03 \mathrm{mg}$ mitochondrial protein and $1 \mathrm{~mm}$-ubiquinol-15. The reaction rate $(v)$ is expressed in ng-atom $\mathrm{O} \mathrm{min}-1$ (mg protein $)^{-1}$.

succinate dehydrogenase-ubiquinone junction (Takemori \& King, 1964). Recently (Trumpower \& Simmons, 1979), this inhibitor has been used to study the mechanism by which succinate dehydrogenase transfers electrons from succinate to ubiquinone and the $b$ and $c_{1}$ cytochromes of the respiratory chain. With ubiquinol-15 as an electron donor it is possible to determine the effect of 2 -thenoyltrifluoroacetone on the $\mathrm{O}_{2}$-reducing part of the alternative oxidase.

The results of the inhibition studies are summarized in Table 2. It is clear that, of the different cytochrome oxidase-mediated oxidase activities tested, only the succinate oxidase activity was inhibited by $1 \mathrm{mM}$-2-thenoyltrifluoroacetone. This agrees with the reported specificity of 2-thenoyltrifluoroacetone for the succinate-ubiquinone junction of the conventional respiratory chain (Garland et al., 1966). With some mitochondrial preparations, the cytochrome oxidase-mediated NADH oxidation was stimulated by $1 \mathrm{mM}-2$ thenoyltrifluoroacetone. Since this effect appears to be unrelated to the activity of the alternative oxidase, it will not be discussed further. That 2-thenoyltrifluoroacetone did not inhibit the cytochrome oxidase-mediated ubiquinol-15 oxidation agrees with the finding of Hatefi et al. (1962) that 2-thenoyltrifluoroacetone does not inhibit ubiquinol-cytochrome $c$ reductase activity. A completely different inhibition pattern was obtained with the alternative oxidase-mediated oxidations. All the activities tested were inhibited more than $80 \%$ by $1 \mathrm{mM}$-2-thenoyltrifluoroacetone, with an almost complete inhibition of the alternative oxidase-mediated ubiquinol-15 oxidation. Because a sensitivity shift was not observed when the alternative oxidase was measured in the presence of cyanide or antimycin A or both, inhibition by 2-thenoyltrifluoroacetone cannot be the result of a cyanide-induced or antimycin A-induced change of an electron carrier. Therefore, it may be concluded that 2-thenoyltrifluoroacetone interacts with a specific electron carrier of the alternative oxidase itself.

A Dixon plot (Dixon, 1953) for the inhibition of the alternative oxidase-mediated ubiquinol-15 oxidation by 2 -thenoyltrifluoroacetone is shown in Fig. 2. The Dixon plot is linear from 10 to $1000 \mu \mathrm{M}$-2-thenoyltrifluoroacetone, a range above which $95 \%$ of the activity is inhibited. Linear Dixon plots reflect simple saturation kinetics and are consistent with the existence of a single binding site for the inhibitor. Alternatively, if there are multiple sites, their affinities for the inhibitor are indistinguishable from each other, as pointed out by 
Trumpower \& Simmons (1979) for the inhibition by 2-thenoyltrifluoroacetone on the succinate-ubiquinone junction of the respiratory chain.

\section{DISCUSSION}

In order to isolate and identify the $\mathrm{O}_{2}$-reducing component of the alternative pathway, it is necessary to find a substrate which bypasses the common electron carriers of both pathways (e.g. succinate-ubiquinone oxidoreductase) and donates electrons at or close to the alternative $\mathrm{O}_{2}$-consuming component. Huq \& Palmer $(1978 b, c)$ discovered that duroquinol is an appropriate substrate for the alternative oxidase of plant mitochondria, whereas Von Jagow \& Bohrer (1975) reported that the oxidation of duroquinol in cyanide-insensitive mitochondria of $N$. crassa is sensitive to antimycin A, suggesting that duroquinol donates electrons to a carrier of the conventional cytochrome chain. In the presence of cyanide, electrons are diverted to the alternative pathway. Our results confirm the finding of Von Jagow \& Bohrer (1975). For this reason, it is obvious that duroquinol is not a suitable substrate for the alternative oxidase of $N$. crassa and that the electron transfer in the presence of duroquinol in plant mitochondria differs from the electron transport in $N$. crassa mitochondria.

Since ubiquinone is thought to be the branchpoint of the two pathways, short-chain ubiquinol homologues were tested as substrates for the alternative oxidase of $N$. crassa. It was found that ubiquinol-15 can be oxidized by both the cytochrome pathway and the alternative pathway, depending on the inhibitor(s) added. The inhibitor sensitivities for the oxidation of all three substrates, succinate, NADH and ubiquinol-15, by the alternative oxidase are similar. Although we did not do experiments with ubiquinone-depleted mitochondria, it is likely that ubiquinol-15 substitutes for endogenously formed ubiquinol, as demonstrated previously with beef heart mitochondria (Ambe \& Crane, 1960). Ubiquinol-15 has been selected from among the short-chain ubiquinol homologues because its redox properties relate very well to those of the physiologically important ubiquinols (Futami et al., 1979). There are, however, some disadvantages in the use of ubiquinone-15: its low solubility in water and its commercial unavailability.

A major step forward in the study of the alternative oxidase was the finding of Schonbaum et al. (1971) that substituted hydroxamic acids specifically inhibit the alternative oxidase activity. The inhibition mechanism of these compounds is not well understood, however. Several mechanisms have been proposed by Schonbaum et al. (1971), among which are chelation of a transition metal ion, most probably ferric ion, and polyfunctional hydrogen bonding. Interaction of hydroxamic acids with an iron-sulphur centre as the possible inhibition mechanism has been favoured in the past (Henry et al., 1973), but Rich et al. (1978) proposed that mechanisms involving either hydrogen bonding or formation of charge-transfer complexes are more feasible. Indeed, Moore \& Rupp (1978) have observed an interaction of salicylhydroxamic acid and semiquinones.

Since both ubiquinone and iron-sulphur centres seem to be required for alternative oxidase activity, and because 2-thenoyltrifluoroacetone is known to disrupt an interaction between ubiquinone and the iron--sulphur centre of succinate dehydrogenase (Nelson et al., 1971), the effect of this compound on the alternative oxidase was tested. The results obtained from those experiments reveal that 2-thenoyltrifluoroacetone is a potent inhibitor of the alternative oxidase activity ( $95 \%$ inhibition of the alternative oxidase-mediated ubiquinol-15 oxidation by $1 \mathrm{~mm}$-2-thenoyltrifluoroacetone), but does not affect the cytochrome oxidase-mediated NADH and ubiquinol-15 oxidase in $N$. crassa mitochondria. Wilson (1971) first reported the effect of 2-thenoyltrifluoroacetone on the alternative oxidase-mediated NADH and malate oxidation of Acer pseudoplatanus mitochondria. He noted the inhibition of NADH and malate oxidation by 2 -thenoyltrifluoroacetone through both pathways, but the inhibitory effect was enhanced by the addition of cyanide to the reaction mixture. Rich et al. (1977) did the same 
experiments with Phaseolus aureus mitochondria, and reported different percentages of inhibition by 2-thenoyltrifluoroacetone of the alternative oxidase-mediated and cytochrome oxidasemediated NADH oxidation, although a difference of only about $30 \%$ was observed. Ziogas \& Georgopoulos (1979) showed that 2-thenoyltrifluoroacetone inhibits NADH oxidation by the alternative pathway but not by the cytochrome pathway in Ustilago maydis mitochondria. With our experiments on NADH and ubiquinol-15 oxidase activities in $N$. crassa mitochondria, it may be concluded that 2-thenoyltrifluoroacetone, at the concentration used, acts on a specific electron carrier which is a component of the alternative oxidase, because there is no inhibition at the NADH-ubiquinone junction and the inhibition of the transfer of electrons from exogenously added ubiquinol-15 to the endogenous ubiquinone pool can be excluded, since the inhibitor does not interfere with the oxidation of ubiquinol-15 by the cyanide-sensitive pathway.

The use of 2-thenoyltrifluoroacetone as an inhibitor of the alternative oxidase is very attractive, since the mechanism of inhibition of the succinate-ubiquinone oxidoreductase by this compound has been recently elucidated. It is believed that 2-thenoyltrifluoroacetone inhibits the reduction of ubisemiquinone to ubiquinol by intercalating between the high potential iron-sulphur centre S-3 of succinic dehydrogenase and the spin-coupled ubisemiquinone pair associated with this centre (Trumpower \& Simmons, 1978, 1979). Hence, the inhibition of the alternative oxidase by 2 -thenoyltrifluoroacetone confirms the importance of ubisemiquinone and the probable involvement of at least one iron-sulphur centre in the activity of the inducible alternative respiratory pathway of $N$. crassa mitochondria.

The results described here have been confirmed with mitochondria of chloramphenicolinduced Moniliella tomentosa (Vanderleyden et al., 1980a), which also oxidize ubiquinol-15 through both pathways. The oxidation through the cytochrome chain is insensitive to $1 \mathrm{~mm}$-2-thenoyltrifluoroacetone but is almost completely blocked through the alternative pathway by this compound.

We thank Hoffman-la-Roche for the generous gift of ubiquinone-15. This research was supported by a grant from 'Het Nationaal Fonds voor Wetenschappelijk Onderzoek' to J.V., and by grant A-6351 from the National Science and Engineering Research Council of Canada to H. B.

\section{REFERENCES}

Ambe, K. S. \& Crane, F. L. (1960). Studies on the electron transport system. XXVI. Specificity of coenzyme $\mathrm{Q}$ and coenzyme $\mathrm{Q}$ derivatives. Biochimica et biophysica acta 43, 30-40.

Bertrand, H. \& Pittenger, T. H. (1969). Cytoplasmic mutants selected from continuously growing cultures of Neurospora crassa. Genetics 61, 643659.

Bertrand, H., McDougall, K. J. \& Pittenger, T. H. (1968). Somatic cell variation during uninterrupted growth of Neurospora in continuous growth tubes. Journal of General Microbiology 50, 337350.

Boveris, A., Ostina, R., Erecinska, M. \& Chance, B. (1971). Reduction of mitochondrial components by durohydroquinone. Biochimica et biophysica acta 245, $1-16$.

Busenherz, G., Bolze, H. J., Bücher, Th., Czoln, R., Garbade, K. H., Meyer-Arendt, E. \& Phleiderer, G. (1953). Diphosphofructose aldolase, phosphoglycerate dehydrogenase, lactic acid dehydrogenase, glycerophosphate dehydrogenase and pyruvate kinase from muscle. Zeitschrift für Naturforschung 39, 59-67.
Dixon, M. (1953). Determination of enzyme-inhibitor constants. Biochemical Journal 55, 170-171.

Futami, A., HuRT, E. \& Hauska, G. (1979). Vectorial redox reactions of physiological quinones. I. Requirement of a minimum length of the isoprenoid side chain. Biochimica et biophysica acta 547, 583-596.

Garland, P. B., Chance, B., Ernster, L., Lee, C. P. \& Wong, E. (1966). Flavoproteins of mitochondrial fatty acids oxidation. Proceedings of the National Academy of Sciences of the United States of America 58, 1696-1702.

Gornall, A. G., Bordawill, C. J. \& David, M. M. (1949). Determination of serum proteins by means of the biuret reaction. Journal of Biological Chemistry 177, 751-766.

Hanssens, L., Von Jagow, G. \& Verachtert, H. (1978). Participation of ubiquinone in the cyanideinsensitive respiration of Moniliella tomentosa. In Functions of Alternative Terminal Oxidases, pp. 47-53. Edited by H. Degn, D. Lloyd \& G. C. Hill. Oxford: Pergamon Press.

Hatefi, Y., HaAvik, A. G. \& Griffiths, D. E. (1962). Studies on the electron transfer system. XLI. 
Reduced coenzyme $\mathrm{Q}\left(\mathrm{QH}_{2}\right)$-cytochrome $c$ reductase. Journal of Biological Chemistry 237, 16811685 .

Henry, M. F., De Troostenbergh, J. C. \& Nyns, E. J. (1973). Reversal by $\mathrm{Fe}^{3+}$ of the inhibition by benzhydroxamic acid of the cyanide-insensitive respiration of Candida lipolytica. Archives internationales de physiologie et de biochemie 81, 971 .

HuQ, S. \& PALmer, J. M. (1978 a). The involvement and possible role of quinone in cyanide-resistant respiration. In Plant Mitochondria, pp. 225-232. Edited by G. Ducet \& C. Lance. Amsterdam: Elsevier/North-Holland Biomedical Press.

HuQ, S. \& PALMER, J. M. $(1978 b)$. Isolation of a cyanide-resistant duroquinol oxidase from Arum maculatum mitochondria. FEBS Letters 95, 217220.

HuQ, S. \& Palmer, J. M. $(1978 c)$. Oxidation of durohydroquinone via the cyanide-insensitive respiratory pathway in higher plant mitochondria. FEBS Letters 92, 317-320.

MOORE, A. L. \& RUPP, H. (1978). The interaction of ubisemiquinones with the iron-sulfur center S-3 of succinate dehydrogenase in plant mitochondria. FEBS Letters 93, 73-77.

Nelson, B. D., Norling, B., Persson, B. \& ERNSTER, L. (1971). Studies with ubiquinonedepleted submitochondrial particles. Biochemical and Biophysical Research Communications 44, 1312-1320.

PALMER, J. M. (1976). The organization and regulation of electron transport in plant mitochondria. Annual Review of Plant Physiology 27, 133-157.

RAMASARMa, T. \& JAYARAMAN, J. (1971). Reversephase chromatographic separation of ubiquinone isoprenologs. Methods in Enzymology 17C, 165169.

RICH, P. R. (1978). Quinol oxidation in Arum maculatum mitochondria and its application in the assay. Solubilization and partial purification of the alternative oxidase. FEBS Letters 96, 252-256.

RICH, P. R. \& BONNER, W. D., JR (1978). The nature and location of cyanide and antimycin resistant respiration in higher plants. In Functions of Alternative Terminal Oxidases, pp. 149-158. Edited by H. Degn, D. Lloyd \& G. C. Hill. Oxford: Pergamon Press.

Rich, P. R., Moore, A. L. \& Bonner, W. D., JR (1977). The effects of bathophenantroline, bathophenantroline-sulphonate and 2-thenoyltrifluoroacetone on Mung-bean mitochondria and submitochondrial particles. Biochemical Journal 162, 205208.

Rich, P. R., Wiegand, N. K., Blum, H., Moore, A. L. \& BONNER, W. D., JR (1978). Studies on the mechanism of inhibition of redox enzymes by substituted hydroxamic acids. Biochimica et biophysica acta 525, 325-337.

RIEsKe, J. S. (1967). Preparation and properties of reduced coenzyme Q-cytochrome $c$ reductase. Methods in Enzymology 10, 239-245.

Schonbaum, G. R., Bonner, W. D., JR, Storey, B. T.
\& BAHR, J. T. (1971). Specific inhibition of the cyanide-insensitive respiratory pathway in plant mitochondria by hydroxamic acids. Plant Physiology 47, 124-128.

Slater, E. C., Colpa-Boonstra, J. P. \& Links, J. (1961). The oxidation of quinols by mitochondrial preparations. In Quinones in Electron Transport, pp. 161-189. Edited by G. E. W. Wolstenholme \& C. M. O'Connors. London: Churchill.

Solomos, T. (1977). Cyanide resistant respiration in higher plants. Annual Review of Plant Physiology 28, 279-297.

TAKEMORI, S. \& KING, T. E. (1964). Coenzyme Q: reversal of inhibition of succinate-cytochrome $c$ reductase by lipophilic compounds. Science 144, 852.

Trumpower, B. L. \& Simmons, Z. (1978). Diminished inhibition of succinate-cytochrome $c$ reductase activity of resolved reductase complex by thenoyltrifluoroacetone in the presence of antimycin. Biochemical and Biophysical Research Communications 82, 289-295.

TRumpower B. L. \& Simmons, Z. (1979). Diminished inhibition of mitochondrial electron transfer from succinate to cytochrome $c$ by thenoyltrifluoroacetone induced by antimycin. Journal of Biological Chemistry 254, 4608-4616.

VANDERLEYDEN, J., VAN DEN EYNDE, E. \& VERACHTERT, H. $(1980 \mathrm{a})$. Nature of the effect of adenosine $5^{\prime}$-monophosphate on the cyanide-insensitive respiration in mitochondria of Moniliella tomentosa. Biochemical Journal 186, 309-316.

Vanderleyden, J., Peeters, C., Verachtert, H. \& BerTRAND, H. $(1980 b)$. Stimulation of the alternative oxidase of Neurospora crassa by nucleoside phosphates. Biochemical Journal 188, 141-144.

VANDERleyden, J., MEyers, M. \& VERAChtert, H. $(1980 c)$. Identification of the quinone species in cyanide-insensitive and cyanide-sensitive mitochondria of Moniliella tomentosa. Biochemical Journal 192, 881-885.

VoGEL, H. J. (1964). Distribution of lysine pathways among fungi: evolutionary implications. American Naturalist 98, 435-466.

VON JAGOW, G. \& BOHRER, C. (1975). Inhibition of electron transfer from ferrocytochrome $b$ to ubiquinone, cytochrome $c_{1}$ and duroquinone by antimycin A. Biochimica et biophysica acta 387 , 409-424.

Weiss, H., Von Jagow, G., Klingenberg, M. \& BÜCHER, T. (1970). Characterization of Neurospora crassa mitochondria prepared with a grind mill. European Journal of Biochemistry 14, 75-82.

WILson, S. B. (1971). Studies on the cyanideinsensitive oxidase of plant mitochondria. FEBS Letters 15, 49-52.

Ziogas, B. N. \& Georgopoulos, S. G. (1979). The effect of carboxin and thenoyltrifluoroacetone on cyanide-sensitive and cyanide-resistant respiration of Ustilago maydis mitochondria. Pesticide Biochemistry and Physiology 11, 208-217. 